\title{
Vacinação para influenza em idosos na pandemia COVID-19: estudo de base populacional em 133 cidades brasileiras
}

\author{
Influenza vaccination in older adults during the COVID-19 \\ pandemic: a population-based study in 133 Brazilian cities
}

\author{
Ana Maria Baptista Menezes (https://orcid.org/0000-0002-2996-9427) ${ }^{1}$ \\ Pedro Curi Hallal (https://orcid.org/0000-0003-1470-6461) ${ }^{1}$ \\ Mariângela Freitas Silveira (https://orcid.org/0000-0002-2861-7139) ${ }^{1}$ \\ Fernando César Wehrmeister (https://orcid.org/0000-0001-7137-1747) \\ Bernardo Lessa Horta (https://orcid.org/0000-0001-9843-412X) ${ }^{1}$ \\ Aluísio Jardim Dornellas de Barros (https://orcid.org/0000-0002-2022-8729) ${ }^{1}$ \\ Fernando Pires Hartwig (https://orcid.org/0000-0003-3729-0710) ${ }^{1}$ \\ Paula Duarte Oliveira (https://orcid.org/0000-0002-5495-3259) ${ }^{1}$ \\ Luís Paulo Vidaletti (https://orcid.org/0000-0002-2840-6841) ${ }^{1}$ \\ Marilia Arndt Mesenburg (https://orcid.org/0000-0001-9598-4193) ${ }^{2}$ \\ Nadege Jacques (https://orcid.org/0000-0002-2204-3330) ${ }^{1}$ \\ Fernando C Barros (https://orcid.org/0000-0001-5973-1746) ${ }^{3}$ \\ Cesar Gomes Victora (https://orcid.org/0000-0002-2465-2180) ${ }^{1}$
}

${ }^{1}$ Universidade Federal de Pelotas. Av. Marechal Deodoro $11603^{\circ}$ piso, Centro. 96020-220 Pelotas RS Brasil.

anamene.epi@gmail.com

${ }^{2}$ Fundação Universidade

Federal de Ciências de

Saúde de Porto Alegre.

Porto Alegre RS Brasil.

${ }^{3}$ Universidade Católica de

Pelotas. Pelotas RS Brasil.

\begin{abstract}
Routine immunization during pandemics can be harmed. This study estimated the influenza vaccination coverage in older adults $d u$ ring the COVID-19 through the EPICOVID-19, a population-based study conducted in 133 cities from the 26 Brazilian states and Federal District. We selected 25 census tracts per city, with probability proportional to the tract's size, ten households by census tract, and one random individual interviewed. A total of 8,265 older adults ( $\geq 60$ years old) were interviewed and asked whether they had been vaccinated against flu in 2020. Vaccination coverage was $82.3 \%$ (95\% CI: 80.1-84.2) with no difference by gender, age, and region; $h i-$ gher vaccination coverage was observed among the wealthiest $(84.7 \%$ versus $80.1 \%$ in the poorest) and among the more educated ( $87.3 \%$ versus $83.2 \%$ less educated); lower coverage among indigenous $(56.9 \%$ versus $>80 \%$ among other ethnic groups). A positive association was identified with the number of comorbidities among men but not among women. Most of the population was vaccinated (97.5\%) in the public health system. The private network was chosen mainly in the South by the wealthiest and more educated. Vaccination coverage was seven percentage points lower than the government target (90\%), and inequalities should be reversed in future campaigns.
\end{abstract}

Key words Vaccination coverage, Influenza virus, Elderly, Coronavirus infections
Resumo Imunizações de rotina durante pandemias podem ser prejudicadas. Este estudo estimou a cobertura vacinal para influenza em idosos durante a COVID-19 através do EPICOVID-19, inquérito populacional realizado em 133 cidades sentinelas dos 26 estados brasileiros e Distrito Federal. Selecionou-se 25 setores censitários por cidade, amostragem proporcional ao tamanho, dez domicílios por setor e uma pessoa por domicílio, aleatoriamente. O quantitativo de 8.265 idosos ( $\geq 60$ anos) foram entrevistados e responderam se haviam sido vacinados contra gripe em 2020. A cobertura foi 82,3\% (IC95\% 80,1; 84,2), sem diferenças por sexo, idade ou região. Maiores coberturas ocorreram nos mais ricos ( $84,7 \%$ versus $80,1 \%$ nos mais pobres) e nos mais escolarizados ( $87,3 \%$ versus $83,2 \%$ nos menos escolarizados). Menor cobertura nos indigenas (56,9\% versus coberturas superiores a $80 \%$ nos demais grupos étnicos). Houve associação positiva com número de comorbidades entre homens, mas não entre mulheres. A maioria vacinou-se na rede pública (97,5\%), sendo a rede privada mais utilizada na região Sul, pelos mais escolarizados e mais ricos. Conclui-se que a cobertura vacinal ficou sete pontos percentuais abaixo da meta governamental (90\%), e que desigualdades devem ser revertidas em futuras campanhas.

Palavras-chave Cobertura vacinal, Virus da influenza, Idosos, Infecções por coronavírus 


\section{Introdução}

Influenza é uma doença respiratória infecciosa de origem viral, com alta morbimortalidade, principalmente em alguns grupos de risco como idosos, crianças, e portadores de doenças crônicas ${ }^{1}$. No Brasil, a vacina para influenza foi incorporada ao Programa Nacional de Imunizações (PNI) desde 1999, com a finalidade de reduzir hospitalizações, complicações e óbitos ${ }^{2,3}$. Apesar da efetividade da vacina ser heterogênea ${ }^{4-6}$, é orientação da Organização Mundial da Saúde (OMS) ${ }^{2}$ e do Ministério da Saúde do Brasil $(\mathrm{MS})^{7}$ realizar a vacinação anualmente, priorizando determinados grupos, incluindo os idosos com 60 anos ou mais ${ }^{8}$. Desde 2017, a meta de cobertura vacinal do PNI consiste em atingir $90 \%$ desta população-alvo'; entretanto, alguns estudos populacionais realizados no país mostram coberturas inferiores, variando de 70 a $78 \%$, nos últimos 10 anos $^{10-13}$.

Frente à pandemia COVID-19, o MS adotou nova estratégia para vacina da gripe em 2020, antecipando a data de início da vacinação para final de março, como também estendendo a vacinação gratuita para outros grupos populacionais, como adultos de 55 a 59 anos e profissionais tais como: portuários, motoristas e cobradores do transporte coletivo, caminhoneiros e profissionais de forças de segurança e salvamento ${ }^{14}$.

Apesar de a vacina contra a influenza não ter eficácia contra o coronavírus, ela pode auxiliar os profissionais da saúde na exclusão do diagnóstico COVID-19, já que os sintomas podem ser parecidos, e reduzir a procura por serviços de saúde ${ }^{14}$.

Cabe lembrar que a população pode sentirse em dúvida em relação à adesão à vacinação: se, por um lado, existe uma recomendação para manter-se em isolamento e evitar aglomerações, por outro lado, a população é chamada para vacinar-se contra gripe, o que pode expor os indivíduos a situações de alto risco de contágio pelo Sars-CoV-2; além da não adesão à vacinação pela própria pandemia, há que considerar outras razões como apontado no artigo de Andrade ${ }^{15}$, tais como: medo de efeitos colaterais da vacina, desconhecimento dos seus benefícios, considerar-se naturalmente imune à gripe, contra-indicação médica, dificuldades financeiras para ir ao posto de saúde, dentre outras.

No ano de 2020 enfrentamos um cenário epidemiológico atípico no país. Várias atividades de cuidados básicos de saúde por parte da população, assim como pelas autoridades de saúde, precisaram de reorganizações e de novas estratégias, sem que houvesse uma adequada avaliação das mudanças ocorridas, já que a urgência era o enfrentamento da pandemia. O estudo EPICOVID-19, sendo em nível nacional e de base populacional, permitiu uma avaliação do novo cenário epidemiológico no ano de 2020.

O objetivo deste artigo foi estimar a cobertura vacinal contra influenza no curso da pandemia COVID-19 em idosos de 60 anos ou mais e sua associação com variáveis sociodemográficas e comorbidades, assim como o percentual de vacinação obtida por meio de provedor privado, na quarta fase do estudo EPICOVID-19.

\section{Métodos}

O EPICOVID-19 16 é um estudo composto por inquéritos sorológicos seriados, delineado para acompanhar a evolução da pandemia do coronavírus no Brasil. A pesquisa foi conduzida em 133 cidades sentinela dos 26 estados brasileiros e Distrito Federal. Essas cidades são as mais populosas de cada uma das 133 regiões intermediárias do Brasil, conforme divisão do Instituto Brasileiro de Geografia e Estatística (IBGE). Em cada uma, 25 setores censitários foram amostrados com probabilidade proporcional ao tamanho e uma lista aleatória de dez domićlios foi fornecida pelo IBGE; em cada domicílio, um dos moradores era sorteado para realizar o teste rápido para detectar anticorpos contra SARS-CoV-2 e responder um questionário. Na quarta fase do estudo, realizada entre os dias 27 e 30 de agosto de 2020, foram coletadas informações sobre a vacinação para influenza, desfecho analisado no presente estudo. Os participantes que responderam sim à pergunta " $\mathrm{O}(\mathrm{a})$ sr.(a) fez a vacina da gripe neste ano?" foram considerados como vacinados para influenza; neste estudo restringiremos os dados de vacina a indivíduos idosos (60 anos ou mais de idade). Calculou-se a cobertura vacinal como o número de pessoas idosas que relataram ter sido vacinadas contra influenza no ano de 2020 dividido pelo total de indivíduos; como o EPICOVID-19 foi um inquéritos de base populacional utilizou-se o termo cobertura vacinal como sinônimo de prevalência ${ }^{17,18}$.

As seguintes características dos indivíduos foram avaliadas: sexo do participante, idade coletada em anos completos (posteriormente categorizada em 60-69, 70-79, $\geq 80$ anos), cor da pele autorreferida (branca, parda, preta, amarela e indígena), região do país (Norte, Nordeste, Sudeste, Sul e Centro-Oeste), índice de bens (em quintis), escolaridade (menos que Fundamental, 
Fundamental, Médio/Graduação incompleta e Graduação completa ou mais) e número de comorbidades (nenhuma, uma, duas, três ou mais). O índice de bens foi criado através de uma análise de componentes principais a partir de uma lista de bens e posses em nível domiciliar, de forma semelhante ao Indicador Econômico Nacional (IEN) ${ }^{19}$. O primeiro componente foi extraído e os domicílios foram ordenados e divididos em quintis, sendo o primeiro quintil representado pelos domicílios $20 \%$ mais pobres da distribuição e o último quintil pelos $20 \%$ mais ricos. Investigou-se o diagnóstico médico autorrelatado das seguintes comorbidades: hipertensão ou pressão alta; diabetes; asma ou bronquite; câncer; doença crônica nos rins; doença do coração; e outra morbidade não mencionada antes.

Foram realizadas análises descritivas para as variáveis consideradas e estimada a cobertura vacinal de acordo com as características dos idosos. As diferenças entre as proporções foram verificadas por meio do teste qui-quadrado ou de tendência linear, considerando-se um nível de significância de 5\%. Para verificar as associações entre as comorbidades e a vacinação contra influenza foram utilizadas razões de cobertura brutas e ajustadas com os respectivos intervalos de confiança de 95\%, através de modelos de regressão de Poisson com variância robusta. Foi utilizado o teste de Mantel-Haenzsel para modificação de efeito entre as variáveis sexo e comorbidades, no modelo de regressão de Poisson bruto, com nível de significância de $10 \%$. No ajuste foram incluídas as variáveis: região do país, idade em categorias, cor da pele, índice de bens e escolaridade.

O estudo EPICOVID-19 foi aprovado pelo Comitê Nacional de Ética em Pesquisa (CONEP). Todos os participantes ou responsáveis, no caso de menores ou pessoas com deficiência, assinaram o termo de consentimento livre e esclarecido. Informações adicionais sobre o EPICOVID-19 podem ser encontradas em publicações anteriores $^{20-22}$.

A taxa geral de resposta do EPICOVID-19 foi de 55\% devido a dificuldades logísticas durante o período de isolamento da pandemia. Em 22\% dos domicílios não foram encontradas pessoas na residência, e em outros $23 \%$ os moradores recusaram o teste para detecção de anticorpos para COVID-19. As perdas e recusas foram substituídas por vizinhos para atingir o total desejado de 33.250 pessoas entrevistadas nas 133 cidades, sendo que dessas, 8.265 (24,9\%) tinham 60 ou mais anos de idade.

\section{Resultados}

As características e cobertura vacinal para influenza entre idosos estão apresentadas na Tabela 1. A cobertura no conjunto das 133 cidades do país foi igual a $82,3 \%$ (IC 95\% 80,1-84,2). Resultados por cidade não são mostrados, pois o número mediano de idosos entrevistados foi de apenas 62 por cidade. Dois terços eram mulheres, 43,3\% eram de cor branca, cerca de 30\% residiam no Nordeste e proporção similar no Sudeste; $1 / 3$ morava em domicílios pobres e mais da metade da amostra tinha escolaridade menor do que o fundamental.

Não houve evidência estatística de diferenças em cobertura conforme sexo, idade ou região do país (Tabela 1). Conforme cor da pele, destaca-se que a cobertura vacinal foi de 56,9\% nos indígenas comparado a coberturas superiores a $80 \%$ nos demais grupos étnicos, mas sem significância estatística. Diferenças estatisticamente significativas foram observadas para a cobertura conforme índice de bens $(\mathrm{p}<0,001)$ e escolaridade $(\mathrm{p}=$ $0,041)$. A variável índice de bens esteve positivamente associada com a cobertura, que variou de $80,1 \%$ no quintil mais pobre a $84,7 \%$ no quintil mais rico. Quanto à escolaridade, os dois grupos extremos apresentaram as maiores coberturas; indivíduos que não completaram o fundamental apresentaram $83,2 \%$ de cobertura, enquanto que naqueles com graduação completa a cobertura foi de $87,3 \%$.

Para a variável cor da pele o valor $\mathrm{p}$ foi igual a 0,056, embora a cobertura entre os 140 indígenas tenha sido de apenas $56,9 \%$, bastante inferior aos demais grupos que apresentaram coberturas superiores a $80 \%$.

Coberturas vacinais de acordo com variáveis sociodemográficas e região, estratificadas por sexo, são mostradas na Figura 1. Na maioria das categorias das variáveis estudadas, as mulheres apresentaram coberturas ligeiramente superiores àquelas observadas entre homens; entretanto, há sobreposição dos intervalos de confiança para homens e mulheres em quase todas as categorias. Em relação à região geográfica, houve modificação de efeito com sexo $(p=0,04)$; entre os homens, a maior cobertura foi nas regiões Sudeste $(84,9 \%)$ e Norte $(83,4 \%)$, enquanto nas mulheres, as maiores coberturas foram nas regiões Centro-Oeste $(84,4 \%)$ e Sudeste $(84,3 \%)$.

$\mathrm{Na}$ Tabela 2 são apresentados os resultados da cobertura vacinal contra influenza para o conjunto dos idosos e estratificada por sexo, de acordo com o número de comorbidades referidas. Para 
Tabela 1. Características da amostra de idosos e cobertura de vacinação contra influenza. Estudo EPICOVID-19, Brasil.

\begin{tabular}{|c|c|c|c|c|c|c|}
\hline Variável & Categoria & $\mathbf{N}$ & $\%$ & $\begin{array}{c}\text { Cobertura } \\
\text { vacinação }(\%)\end{array}$ & IC 95\% & Valor $\mathbf{p}$ \\
\hline \multirow[t]{5}{*}{ Região do país } & Norte & 984 & 11,9 & 79,1 & $74,6-83,0$ & 0,129 \\
\hline & Nordeste & 2.510 & 30,3 & 80,5 & $77,7-83,1$ & \\
\hline & Sudeste & 2.452 & 29,6 & 84,5 & $80,3-87,9$ & \\
\hline & Sul & 1.518 & 18,3 & 79,9 & $76,8-82,6$ & \\
\hline & Centro-Oeste & 828 & 10,0 & 81,1 & $75,5-85,7$ & \\
\hline \multirow[t]{2}{*}{ Sexo } & Masculino & 3.053 & 36,8 & 81,7 & $78,0-84,8$ & 0,658 \\
\hline & Feminino & 5.239 & 63,2 & 82,6 & $79,9-85,0$ & \\
\hline \multirow[t]{3}{*}{ Idade } & $60-69$ & 4.557 & 55,0 & 82,0 & $79,1-84,5$ & $0,123^{*}$ \\
\hline & $70-79$ & 2.671 & 32,2 & 83,2 & $79,4-86,5$ & \\
\hline & $80+$ & 1.064 & 12,8 & 81,3 & $74,5-86,6$ & \\
\hline \multirow[t]{5}{*}{ Cor da pele } & Branca & 3.423 & 43,3 & 82,1 & $78,6-85,1$ & 0,056 \\
\hline & Parda & 3.041 & 38,4 & 83,1 & $79,8-86,0$ & \\
\hline & Preta & 1.059 & 13,4 & 80,5 & $73,7-85,9$ & \\
\hline & Amarela & 246 & 3,1 & 87,1 & $78,3-92,6$ & \\
\hline & Indígena & 140 & 1,8 & 56,9 & $30,6-79,8$ & \\
\hline \multirow[t]{5}{*}{ Índice de bens (quintis) } & Mais pobre & 2.566 & 30,9 & 80,1 & $76,0-83,6$ & $<0,001^{\star}$ \\
\hline & $2^{\circ}$ & 1.693 & 20,4 & 81,1 & $75,5-85,6$ & \\
\hline & $3^{\circ}$ & 1.346 & 16,2 & 81,2 & $74,8-86,2$ & \\
\hline & $4^{\circ}$ & 1.434 & 17,3 & 85,3 & $80,7-88,9$ & \\
\hline & Mais rico & 1.253 & 15,1 & 84,7 & $79,7-88,7$ & \\
\hline \multirow[t]{4}{*}{ Escolaridade } & Menos que Fundamental & 4.178 & 51,6 & 83,2 & $80,1-85,8$ & 0,041 \\
\hline & Fundamental & 1.441 & 17,8 & 77,1 & $70,3-82,8$ & \\
\hline & Médio & 1.650 & 20,4 & 82,1 & $77,8-85,8$ & \\
\hline & Graduação completa & 823 & 10,2 & 87,3 & $83,1-90,6$ & \\
\hline Total & & 8.265 & 100 & 82,3 & $80,1-84,2$ & \\
\hline
\end{tabular}

IC - intervalo de confiança; Valores p do teste qui-quadrado de heterogeneidade; ${ }^{*}$ Teste qui-quadrado de tendência linear.

Fonte: elaboração própria a partir dos dados do Estudo EPICOVID-19, Brasil.

as mulheres não houve diferença estatisticamente significativa para a razão de cobertura ajustada $(\mathrm{p}=0,278)$. Nos homens observaram-se maiores coberturas entre aqueles com duas $(\mathrm{RP}=1,14$; IC95\%: 1,$02 ; 1,19)$ e três ou mais comorbidades $(\mathrm{RP}=1,19 ; \mathrm{IC} 95 \%: 1,07 ; 1,31)$, sendo $19 \%$ maior nestes últimos comparados ao grupo sem esta condição presente $(\mathrm{p}=0,007)$.

Na Figura 2 é apresentado o percentual de obtenção da vacina em provedor privado de saúde, segundo variáveis sociodemográficas. Para o conjunto dos idosos, quase a totalidade $(97,5 \%)$ obteve a vacina na rede pública. Entre aqueles que a adquiriram por meio de provedor privado, não houve diferença nos percentuais quanto ao sexo e faixas etárias ( $\mathrm{p}>0,05)$. Entretanto, 7,7\% dos idosos da região Sul obtiveram a vacina na rede privada, proporção cinco vezes maior do que a observada na região Sudeste $(1,4 \%)$; os mais escolarizados $(7,5 \%)$ e aqueles pertencentes ao maior quintil de riqueza (5,3\%) apresentaram maior percentual de vacinação na rede privada.

\section{Discussão}

A cobertura vacinal para influenza em indivíduos idosos durante a pandemia COVID-19 nas cidades sentinela do estudo EPICOVID-19 foi de 82,3\% (IC 95\% 80,1; 84,2). Não foram observadas diferenças por sexo, idade ou região do país, mas foram observadas maiores coberturas entre indivíduos mais ricos e mais escolarizados; entre os indígenas a cobertura foi menor do que entre os demais grupos étnicos, mas sem significância estatística. Entre os homens, mas não entre as mulheres, observou-se associação direta da cobertura com o número de comorbidades, mesmo 

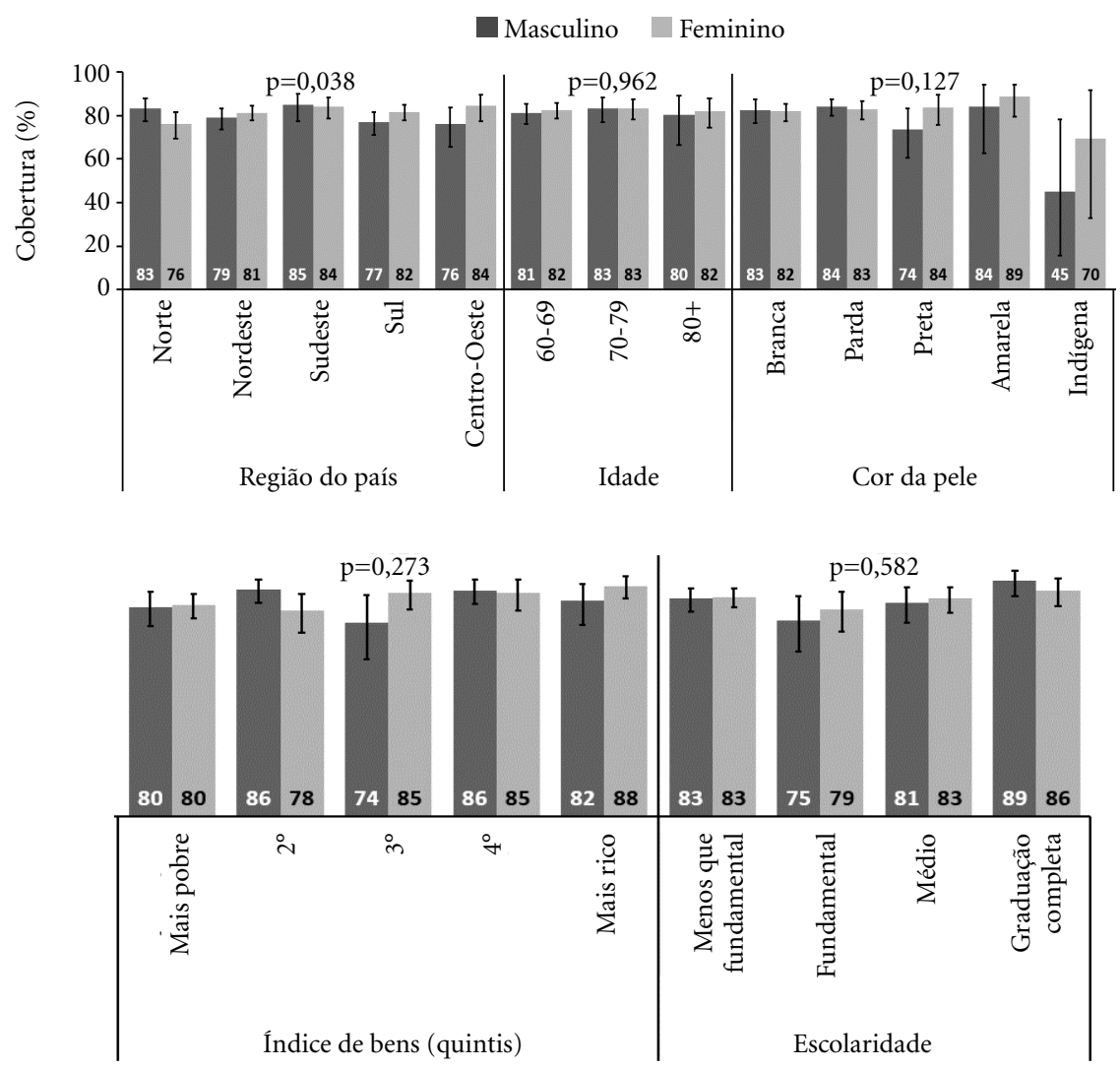

Figura 1. Cobertura vacinal em idosos segundo características sociodemográficas, estratificada por sexo. Estudo EPICOVID-19, Brasil (as barras representam o intervalo de confiança de 95\% para proporções).

Nota: valor p apresentado se refere ao teste de modificação de efeito da variável com sexo.

Fonte: elaboração própria a partir dos dados do Estudo EPICOVID-19, Brasil.

ajustando para fatores de confusão. A maioria da população obteve a vacina na rede pública $(97,5 \%)$, sendo que dentre os que obtiveramna pela rede privada, o maior percentual foi na região Sul, dentre os mais escolarizados e mais ricos.

A meta do governo federal para a cobertura vacinal para influenza em idosos era atingir $80 \%$ no período de 2008-2016; porém, estudos de base populacional realizados em alguns municípios de São Paulo e no Sul do Brasil mostraram coberturas variando de $71 \%{ }^{18}$ a $78 \%{ }^{11-13}$. Desde 2017 , esta meta mudou para atingir $90 \%$ da população idosa ${ }^{9}$. Segundo dados do Sistema de Informação do Programa Nacional de Imunização (SI-PNI) ${ }^{24}$, a razão entre doses administradas e a estimativa populacional de idosos mostrou-se estável em um patamar ao redor de 100\% entre 2016 e 2019, havendo aumentado mais de 20 pontos percentuais de 2019 para 2020, quando atingiu $120,7 \%$. É importante ressaltar a necessidade da atualização contínua dos dados censitários frente à velocidade do envelhecimento da população brasileira; as altas taxas de coberturas vacinais nos últimos anos podem ser devido ao fato da população utilizada para o cálculo estar subestimada $^{25}$. Independentemente da provável defasagem entre o número de doses administradas e a estimativa populacional, o aumento observado em 2020 deve estar relacionado à intensificação da campanha nacional de vacinação contra a gripe nas unidades sanitárias e através dos meios de comunicação social. Apesar de toda campanha realizada para que a população idosa fosse vaci- 
Tabela 2. Comorbidades, cobertura vacinal e razões de coberturas brutas e ajustadas. Estudo EPICOVID-19, Brasil.

\begin{tabular}{|c|c|c|c|c|c|c|c|c|}
\hline Variável & $\mathbf{N}$ & $\%$ & $\begin{array}{c}\text { Cobertura } \\
\text { vacinal } \\
(\%)\end{array}$ & $\begin{array}{c}\text { Valor } \\
\mathbf{p}\end{array}$ & $\begin{array}{c}\text { Razão de } \\
\text { cobertura bruta } \\
(\text { IC 95\%) }\end{array}$ & $\begin{array}{c}\text { Valor } \\
\mathbf{p}\end{array}$ & $\begin{array}{c}\text { Razão de cobertura } \\
\text { ajustada* } \\
\text { (IC 95\%) }\end{array}$ & $\begin{array}{c}\text { Valor } \\
\mathbf{p}\end{array}$ \\
\hline \multicolumn{9}{|c|}{ Comorbidades } \\
\hline Nenhuma & 1.848 & 22,9 & 79,5 & $<0,001$ & 1 & 0,093 & 1 & 0,076 \\
\hline 1 & 2.702 & 33,5 & 81,2 & & $1,02(0,95 ; 1,10)$ & & $1,02(0,95 ; 1,10)$ & \\
\hline 2 & 2.122 & 26,3 & 85,9 & & $1,08(1,01 ; 1,15)$ & & $1,09(1,02 ; 1,17)$ & \\
\hline $3+$ & 1.400 & 17,3 & 82,6 & & $1,04(0,96 ; 1,13)$ & & $1,04(0,96 ; 1,08)$ & \\
\hline \multicolumn{9}{|l|}{ Mulheres } \\
\hline \multicolumn{9}{|c|}{ Comorbidades } \\
\hline Nenhuma & 1.018 & 55,1 & 81,9 & 0,025 & 1 & 0,298 & 1 & 0,278 \\
\hline 1 & 1.699 & 62,9 & 82,0 & & $1,00(0,92 ; 1,09)$ & & $1,00(0,92 ; 1,09)$ & \\
\hline 2 & 1.407 & 66,3 & 86,2 & & $1,05(0,97 ; 1,14)$ & & $1,06(0,98 ; 1,15)$ & \\
\hline $3+$ & 973 & 69,5 & 80,0 & & $0,98(0,87 ; 1,09)$ & & $0,98(0,88 ; 1,10)$ & \\
\hline \multicolumn{9}{|l|}{ Homens } \\
\hline \multicolumn{9}{|c|}{ Comorbidades } \\
\hline Nenhuma & 830 & 44,9 & 75,9 & $<0,001$ & 1 & 0,019 & 1 & 0,007 \\
\hline 1 & 1.003 & 37,1 & 80,1 & & $1,06(0,94 ; 1,19)$ & & $1,06(0,94 ; 1,19)$ & \\
\hline 2 & 715 & 33,7 & 85,4 & & $1,13(1,00 ; 1,27)$ & & $1,14(1,02 ; 1,27)$ & \\
\hline $3+$ & 427 & 30,5 & 88,3 & & $1,16(1,05 ; 1,29)$ & & $1,19(1,07 ; 1,31)$ & \\
\hline
\end{tabular}

Fonte: elaboração própria a partir dos dados do Estudo EPICOVID-19, Brasil.

nada para gripe, nossos resultados do inquérito populacional sugerem que a cobertura em 2020 foi sete pontos percentuais inferior à meta de $90 \%$. A cobertura de $83 \%$ pode ser considerada como satisfatória, devido ao fato de que diante da pandemia houve forte recomendação para que os idosos, que constituem grupo de maior risco para COVID-19, permanecessem em casa distanciando-se de amigos, parentes, vizinhos e evitassem eventos sociais e religiosos como forma de prevenir a doença.

Mulheres geralmente são as que mais procuram serviços de saúde, adotam medidas preventivas e seguem mais indicações relacionadas à saúde ${ }^{26,27}$. Nossos resultados mostraram uma diferença de 0,9 ponto percentual a favor das mulheres, embora sem significância estatística. A falta de diferenças por sexo é consistente com outros estudos brasileiros sobre o tema ${ }^{12,13,15,23}$. Tampouco observamos diferenças entre os três grupos etários de idosos, o que é consistente com alguns estudos ${ }^{12,15,23}$, embora outros estudos tenham encontrado maiores coberturas vacinais em pessoas do grupo etário mais velho ${ }^{13,28}$; acredita-se que frente ao panorama da pandemia COVID-19, em que um dos grupos prioritários da campanha para gripe eram idosos de 60 anos ou mais, a cobertura se distribuiu de forma mais uniforme neste grupo do que em outros estudos realizados em momentos sem pandemia.

Nossos resultados mostraram coberturas similares em relação às regiões do país, variando entre $79,1 \%$ na região Norte e $84,5 \%$ no Sudeste, enquanto que os dados do SI-PNI ${ }^{24}$ sugerem maiores coberturas nas cidades da região Nordeste. $\mathrm{O}$ estudo de Andrade ${ }^{15}$, avaliando uma amostra nacional representativa de indivíduos com 60 anos ou mais, mostrou uma cobertura vacinal contra influenza no país de $72,6 \%$ (IC $95 \% 71,1 ; 74,1)$, com diferentes coberturas vacinais por regiões $(\mathrm{p}<0,001)$, sendo o Norte e Nordeste as regiões de menor cobertura (71,2\% e $66,6 \%$, respectivamente), e o Sul com a maior cobertura $(78,7 \%)$. Dentre as razões apontadas pela população para não se vacinar, no estudo de Andrade, "não saber que era necessário fazer a vacina contra influenza” esteve entre os cinco principais motivos, com percentuais de $7,4 \%$ na região Norte, $4,4 \%$ no Nordeste e $2,1, \%$ na região $\mathrm{Sul}^{15}$. Na Pesquisa Nacional de Saúde de 2013, foi analisado um recorte para amostra de idosos hipertensos, sendo que nesses a maior cobertura vacinal foi na região Sudeste e Sul, o que foi atribuído à melhor posição socioeconômica com um possível maior acesso aos serviços de saúde e melhor aconselhamento em relação à vacinação ${ }^{29}$. 


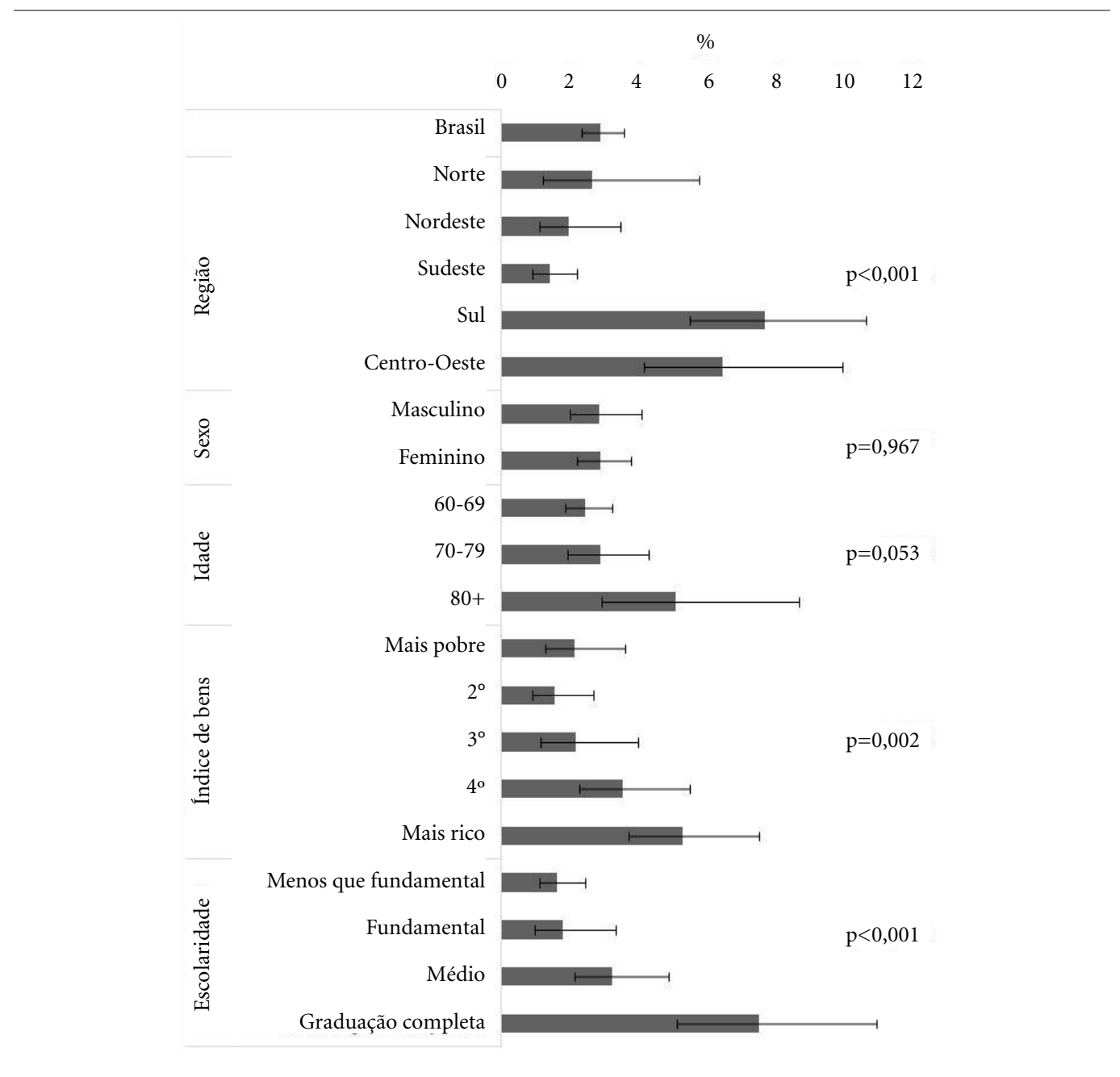

Figura 2. Aquisição da vacina contra influenza em provedor privado de saúde. Estudo EPICOVID-19, Brasil (as barras representam o intervalo de confiança de $95 \%$ para proporções).

Fonte: elaboração própria a partir dos dados do Estudo EPICOVID-19, Brasil.

Outro aspecto a ser considerado na imunização contra influenza é a sazonalidade da doença nas diferentes regiões do país: ao Sul há uma pronunciada sazonalidade com a maior ocorrência de influenza nos meses de inverno, ou seja, no período pós campanha vacinal, sendo que ao norte do país o pico da doença ocorre mais no período que antecede a campanha vacinal ${ }^{30}$. É possível que no estudo EPICOVID-19, durante o ano de 2020, não se tenha observado diferenças significativas na cobertura vacinal por regiões devido à intensificação da campanha vacinal em todo país, e pelo fato de que a pandemia COVID-19 era o que mais amedrontava a população, podendo inclusive manifestar-se com sintomas respiratórios semelhantes à influenza.
A relação entre imunização contra influenza e condições socioeconômicas tem sido um dos pontos controversos na literatura. Estudos realizados no estado de São Paulo apontaram tanto maior cobertura em pessoas com menor escolaridade ${ }^{10,31}$, como ausência de associação entre cobertura e escolaridade ${ }^{28}$. Em outro estudo com idosos ao Sul do Brasil, Neves et al..$^{23}$ verificaram prevalência de vacinação contra influenza $20 \%$ maior naqueles com nível econômico mais alto, sem associação significativa para escolaridade.

Importante ressaltar que a maioria dos estudos mostra a presença e o número de comorbidades como importantes fatores para adesão à vacinação contra influenza ${ }^{11,12,23}$. Em nosso estudo, observamos maior cobertura vacinal entre 
os homens, que relataram maior número de comorbidades, mas não entre mulheres. No estudo de Bacurau $^{32}$ foram observadas diferenças com significância estatística, no sexo feminino, para as comorbidades asma, artrite ou reumatismo, doenças no pulmão ou doença pulmonar obstrutiva crônica e insuficiência renal, e, no sexo masculino, apenas para a comorbidade acidente vascular cerebral; hipertensão arterial diabetes mellitus e doenças do coração não mostraram significância estatística conforme o sexo. O Estudo Saúde, Bem-estar e Envelhecimento (SABE, 2015), com amostra de base populacional de idosos do município de São Paulo, não mostrou diferença estatisticamente significativa para número de comorbidades $(p=0,642)$, sendo que para as comorbidades avaliadas separadamente, tais como hipertensão arterial, diabetes mellitus, doença cardiovascular, doença pulmonar crônica e queda nos últimos 12 meses também não mostraram significância estatística. A presença de depressão conforme o autorrelato foi a única comorbidade que mostrou menor cobertura atingindo a significância estatística $(\mathrm{p}=0,031)^{13}$. Analisando cada uma das comorbidades autorreferidas no inquérito EPICOVID-19 e os seus números, estratificadas por sexo, encontrou-se maior prevalência no sexo masculino para duas ou três ou mais comorbidades no que se refere à hipertensão arterial sistêmica, diabetes, câncer, doença renal e cardíaca, comparado à asma e outras doenças não especificadas, no sexo feminino; sendo assim, acredita-se que a maior cobertura da vacinal nos homens possa ter ocorrido pelo fato dos mesmos referirem doenças mais graves do que as mulheres, o que levaria a maior procura por serviços de saúde e, consequentemente, maior imunização para influenza.

A literatura nacional é unânime quanto à rede pública ser a principal fonte para obtenção da vacina ${ }^{23,31}$, como também foi encontrado no presente estudo, em que menos de $3 \%$ das vacinas foram adquiridas no setor privado. O Brasil está dentre os países que implementaram a vacinação contra influenza desde 1999, com campanhas anuais sazonais e vacinação gratuita para os grupos prioritários ${ }^{14}$. Apesar da recomendação da OMS para que todos países tenham programas nacionais de imunização para influenza ${ }^{33}$ nem todos seguem esta recomendação, sendo que em alguns a mesma não é oferecida sem custo havendo menores coberturas vacinais ${ }^{34}$.

No nosso estudo, o setor privado foi mais frequentemente utilizado na região Sul e, em nível nacional, entre idosos com melhor condição socioeconômica, o que reforça a importância da gratuidade e disponibilidade da vacina.

Especificamente durante a pandemia COVID-19, autoridades mundiais em saúde têm alertado a população sobre os possíveis riscos da falta da vacinação para influenza e de outras vacinas $^{35-37}$. As medidas de isolamento adotadas para mitigar a pandemia podem influenciar negativamente na busca da vacina, principalmente no grupo dos idosos, população essa também de maior risco para COVID-19. Estudo piloto na Austrália ${ }^{38}$ mostrou que estratégias como vacinação para influenza no próprio domicílio podem ser adotadas com sucesso durante uma pandemia.

Algumas limitações deste estudo devem ser mencionadas. A mais importante é a falta de confirmação do status vacinal por meio de carteira ou comprovante de vacinação. No entanto, alguns estudos de validação tanto na população em geral como em grupos específicos mostram alta sensibilidade para o autorrelato (ao redor de $97 \%$ ) ${ }^{39,40}$. Considerando essa alta sensibilidade do autorrelato e o pouco tempo decorrido entre o inquérito e a vacinação é pouco provável a ocorrência do viés de memória, embora não possa ser excluído. O EPICOVID-19 foi restrito a zonas urbanas, o que não nos permite avaliar a cobertura vacinal na zona rural, onde vivem $14,2 \%$ dos brasileiros idosos $^{41}$. Além disso, as cidades sentinela amostradas tendem a ser maiores, mais desenvolvidas e mais bem equipadas com serviços de saúde do que outras áreas urbanas. Indivíduos de cor de pele branca estiveram sub-representados em nossa amostra comparado com a população nacional, provavelmente pela existência de mais recusas em determinados locais de moradia. Ainda, o índice de desenvolvimento humano (IDH) médio das 133 cidades incluídas é superior ao das 5.437 cidades não incluídas, o que pode ter superestimado a cobertura vacinal. Sobre os dados oficiais de cobertura vacinal conforme o IDH dos municípios $^{24,42}$, observa-se uma maior cobertura vacinal contra influenza conforme o quintil do IDH (118\% de cobertura vacinal no maior quintil de IDH e 109,9\% de cobertura no menor quintil de IDH).

Por outro lado, destacam-se como pontos positivos do inquérito EPICOVID-19 o tamanho da amostra do estudo, a abrangência de extensa área do país e a informação sobre a cobertura vacinal para influenza na vigência de uma pandemia como a COVID-19, em que o cenário epidemiológico é singular, sem que tenham sido encontrados outros estudos no país sobre o tema. 
A vacinação para influenza é um programa custo-efetivo, mas para isto ser alcançado há que ter boa adesão da população-alvo. Apesar da cobertura vacinal para influenza em idosos durante a COVID-19 ter sido satisfatória, algumas desigualdades como menor cobertura nos menos escolarizados e nos menos ricos merecem especial atenção em futuras campanhas vacinais. Ainda destacamos os indígenas com bem menor cobertura do que os demais grupos étnicos, apesar da ausência de significância estatística. Vale a reflexão sobre como superar estas desigualdades e planejar as imunizações de acordo com o perfil epidemiológico do momento. As campanhas a favor da vacinação são importantes e parecem estar contribuindo para uma melhor adesão da população. Entretanto, naqueles mais vulneráveis, em quem a cobertura é menor, talvez deva ser pensado em busca ativa e visitas domiciliares através de agentes comunitários de saúde, ou outros profissionais de saúde, visando uma cobertura universal e promoção de um envelhecimento saudável.

\section{Colaboradores}

AMB Menezes foi responsável pela concepção, análise e redação do artigo. PC Hallal é o coordenador do estudo EPICOVID-19. CG Victora, FC Wehrmeister e PD Oliveira ajudaram na análise e discussão dos resultados. Participaram da revisão crítica do artigo: CG Victora, PC Hallal, MF Silveira, FC Wehrmeister, BL Horta, AJD Barros, FP Hartwig, PD Oliveira, LP Vidaletti, MA Mesenburg, N Jacques, FC Barros. Revisão do artigo: Todos os autores. Aprovação final do artigo para publicação e responsabilidade pública por todos aspectos da pesquisa: todos autores.

\section{Agradecimento}

Ao IBOPE, empresa responsável pela coleta de dados do estudo EPICOVID-19.

\section{Financiamento}

O estudo foi financiado pelo Ministério da Saúde do Brasil, Instituto Serrapilheira, Associação Brasileira de Saúde Coletiva (ABRASCO), JBS S.A. Iniciativa Fazer Bem Faz Bem, FAPESP e Todos pela Saúde. 


\section{Referências}

1. World Health Organization (WHO). Immunization, Vaccines and Biologicals - Influenza vaccines; Geneva: WHO; c2020. [cited 2020 Dec 16]. Available from: https://www.who.int/immunization/research/development/influenza/en/.

2. World Health Organization (WHO). Evaluation of influenza vaccine effectiveness: a guide to the design and interpretation of observational studies. Geneva: WHO; 2017. [cited 2020 Dec 16]. Available from: https://apps.who.int/iris/bitstream/handle/10665/ 255203/9789241512121-eng.pdf.

3. Fundação Oswaldo Cruz (Fiocruz). Caminhos da vacinação contra o vírus influenza no Brasil. Rio de Janeiro: Fiocruz;2005 [acessado $2020 \mathrm{dez} 16$ ]. Disponível em: https://portal.fiocruz.br/noticia/caminhosda-vacinacao-contra-o-virus-influenza-no-brasil.

4. Cruzeta AP, Schneider IJ, Traebert J. Impact of seasonality and annual immunization of elderly people upon influenza-related hospitalization rates. Int $J$ Infect Dis 2013;17(12):e1194-1197.

5. Sofia Arriola C, El Omeiri N, Azziz-Baumgartner E, Thompson MG, Sotomayor-Proschle V, Fasce RA, Von Horoch M, Enrique Carrizo Olalla J, Aparecida Ferreira de Almeida W, Palacios J, Palekar R, Couto P, Descalzo M, María Ropero-Álvarez A. Influenza vaccine effectiveness against hospitalizations in children and older adults-Data from South America, 20132017. A test negative design. Vaccine X 2019;3:100047.

6. Heo JY, Song JY, Noh JY, Choi MJ, Yoon JG, Lee SN, Cheong HJ, Kim WJ. Effects of influenza immunization on pneumonia in the elderly. Hum Vaccin Immunother 2018;14(3):744-749.

7. AcVida [Internet]. Vacinação de idosos no Brasil: sua importância e o calendário; c2020 [acessado $2020 \mathrm{dez}$ 20]. Disponível em: https://acvida.com.br/familias/ vacinacao-de-idosos-no-brasil/.

8. Brasil. Ministério da Saúde (MS). Informe técnico: 21 a Campanha Nacional de Vacinação contra a Influenza 2019; Brasília: MS;2019 [acessado $2020 \mathrm{dez}$ 11]. Disponível em: https://portalarquivos2.saude. gov.br/images/pdf/2019/marco/01/Informe-Cp-Influenza-29-02-2019 -final.pdf.

9. Brasil. Ministério da Saúde (MS). Informe Técnico da 19a Campanha Nacional de Vacinação contra a Influenza. Brasília: MS;2017 [acessado 2020 dez 11]. Disponível em: http://pni.datasus.gov.br/ sipni/03\%2003\%202017\%20Informe_Cp_Influenza\%20_\%20final.pdf.

10. Pinto CJM, Pereira EHR, Teodoro CM, Becari RA, Assis VGd, Ferrari JC, Hoehne EL. Vaccination against influenza in elderly people: factors associated with acceptance and refusal of the vaccine. Rev Soc Bras Med Trop 2019;52.

11. Sato AP, Antunes JL, Moura RF, de Andrade FB, Duarte YA, Lebrao ML. Factors associated to vaccination against influenza among elderly in a large Brazilian metropolis. PLoS One 2015;10(4):e0123840.

12. Francisco PMSB, Borim FSA, Neri AL. Vacinação contra influenza em idosos: dados do FIBRA, Campinas, São Paulo, Brasil. Cien Saude Colet 2015;20(12):37753786.
13. Sato APS, Andrade FB, Duarte YAO, Antunes JLF. Vaccine coverage and factors associated with influenza vaccination in the elderly in the city of Sao Paulo, Brazil: SABE Study 2015. Cad Saude Publica 2020;36 (Suppl. 2):e00237419.

14. Ministério da Saúde (MS). Informe Técnico: 22a Campanha Nacional de Vacinação contra a Influenza 2020. [acessado 2021 abr 22]. Disponível em: https:// www.saude.go.gov.br/files/imunizacao/influenza/InformeTecnicoInfluenza.2020.pdf.

15. Andrade FB, Sayuri Sato AP, Moura RF, Ferreira Antunes JL. Correlates of influenza vaccine uptake among community-dwelling older adults in Brazil. Hum Vaccin Immunother 2017; 13(1):103-110.

16. Hallal PC, Barros FC, Silveira MF, Barros AJD, Dellagostin OA, Pellanda LC, Struchiner CJ, Burattini MN, Hartwig FP, Menezes AMB, Horta BL, Victora CG. EPICOVID19 protocol: repeated serological surveys on SARS-CoV-2 antibodies in Brazil. Cien Saude Colet 2020; 25(9):3573-3578.

17. Moraes JCd, Ribeiro MCSdA. Desigualdades sociais e cobertura vacinal: uso de inquéritos domiciliares. Rev Bras Epidemiol 2008; 11:113-124.

18. Barata RB, Moraes JC, Antonio PR, Dominguez M. Immunization coverage survey: empirical assessment of the cluster sampling method proposed by the World Health Organization. Rev Panam Salud Publica 2005; 17(3):184-190.

19. Barros AJ, Victora CG. A nationwide wealth score based on the 2000 Brazilian demographic census. Rev Saude Publica 2005; 39(4):523-529.

20. Hallal PC, Hartwig FP, Horta BL, Silveira MF, Struchiner CJ, Vidaletti LP, Neumann NA, Pellanda LC, Dellagostin OA, Burattini MN, Victora GD, Menezes AMB, Barros FC, Barros AJD, Victora CG. SARSCoV-2 antibody prevalence in Brazil: results from two successive nationwide serological household surveys. Lancet Glob Health 2020; 8(11):e1390-e1398.

21. Horta BL, Silveira MF, Barros AJD, Barros FC, Hartwig FP, Dias MS, Menezes AMB, Hallal PC, Victora CG. Prevalence of antibodies against SARS-CoV-2 according to socioeconomic and ethnic status in a nationwide Brazilian survey. Rev Panam Salud Publ 2020; 44:e135.

22. Epicovid19brasil.org. EpiCovid19 - Pesquisa Epidemiológica Covid-19; c2020 [acessado 2021 abr 22]. Disponível em: http://www.epicovid19brasil.org/.

23. Neves RG, Duro SM, Tomasi E. Influenza vaccination among elderly in Pelotas-RS, Brazil, 2014: a population-based study. Epidemiol Serv Saude 2016; 25(4):755-766.

24. DATASUS. SIPNI - Sistema de Informações do Programa Nacional de Imunizações; Brasília: Ministério da Saúde; 2020 [acessado 2020 nov 20]. Disponível em: http://sipni.datasus.gov.br/si-pni-web/faces/inicio.jsf.

25. Azambuja HCS, Carrijo MF, Martins TCR, Luchesi BM. The impact of influenza vaccination on morbidity and mortality in the elderly in the major geographic regions of Brazil, 2010 to 2019. Cad Saude Publica 2020; 36(Suppl. 2):e00040120. 
26. Dilelio AS, Tomasi E, Thume E, Silveira DS, Siqueira FC, Piccini RX, Silva SM, Nunes BP, Facchini LA. Patterns in the use of outpatient care in Brazil by patients treated through the Brazilian Unified National Health System, private health insurance, and out-of-pocket medical care. Cad Saude Publica 2014; 30(12):25942606.

27. Nunes BP, Flores TR, Garcia LP, Chiavegatto ADF, Thume E, Facchini LA. Time trend of lack of access to health services in Brazil, 1998-2013. Epidemiol Serv Saude 2016; 25(4):777-787.

28. Sato APS, Antunes JLF, Lima-Costa MFF, Andrade FB. Influenza vaccine uptake among older adults in Brazil: Socioeconomic equality and the role of preventive policies and public services. J Infect Public Health 2020; 13(2):211-215.

29. Bacurau AGM, Francisco P. Reasons for non-vaccination against influenza among older adults with hypertension in Brazil: a cross-sectional study. Sao Paulo Med J 2020; 138(4):322-325.

30. Alonso WJ, Viboud C, Simonsen L, Hirano EW, Daufenbach LZ, Miller MA. Seasonality of influenza in Brazil: a traveling wave from the Amazon to the subtropics. Am J Epidemiol 2007; 165(12):1434-1442.

31. Monteiro CN, Gianini RJ, Stopa SR, Segri NJ, Barros MBA, Cesar CLG, Goldbaum M. Vaccination coverage and use of the Brazilian Health System for vaccination against influenza and pneumonia in adults and elderly with self-reported diabetes, municipality of Sao Paulo, 2003, 2008 and 2015. Epidemiol Serv Saude 2018; 27(2):e2017272.

32. Bacurau AGM, Francisco P. Prevalence of influenza vaccination in elderly Brazilian with chronic diseases. Cad Saude Publica 2019; 35(4):e00230518.

33. World Health Organization (WHO). Global action plan for influenza vaccines; [cited $2021 \mathrm{Apr} 22$ ]. Geneva: WHO;2021. Available from: https://www.who. int/influenza_vaccines_plan/en/.

34. Principi N, Camilloni B, Esposito S. Influenza immunization policies: Which could be the main reasons for differences among countries? Hum Vaccin Immunother 2018; 14(3):684-692.

35. Gostin LO, Salmon DA. The Dual Epidemics of COVID-19 and influenza: vaccine acceptance, coverage, and mandates. JAMA 2020; 324(4):335-336.

36. Odone A, Bucci D, Croci R, Riccò M, Affanni P, Signorelli C. Vaccine hesitancy in COVID-19 times. An update from Italy before flu season starts. Acta Biomed 2020; 91(3):e2020031.

37. Grohskopf LA, Alyanak E, Broder KR, Blanton LH, Fry AM, Jernigan DB, Atmar RL. Prevention and Control of Seasonal Influenza with Vaccines: Recommendations of the Advisory Committee on Immunization Practices - United States, 2020-21 Influenza Season. MMWR Recomm Rep 2020; 69(8):1-24.
38. Nisbet LC, Cobbledick AM, Smith TE, Bryant PA, Lawrence J. Opportunistic influenza vaccination in the home: broadening access in isolated times. Arch Dis Child 2020; Oct 27:archdischild-2020-320273. Ahead of print.

39. Zimmerman RK, Raymund M, Janosky JE, Nowalk MP, Fine MJ. Sensitivity and specificity of patient self-report of influenza and pneumococcal polysaccharide vaccinations among elderly outpatients in diverse patient care strata. Vaccine 2003; 21(1314):1486-1491.

40. Smith R, Hubers J, Farraye FA, Sampene E, Hayney MS, Caldera F. Accuracy of Self-Reported Vaccination Status in a Cohort of Patients with Inflammatory Bowel Disease. Dig Dis Sci 2020; 29:1-7.

41. Instituto Brasileiro de Geografia e Estatística (IBGE). Pesquisa nacional de saúde, 2019 [base de dados]. Brasília: IBGE;2020. [acessado 2021 abr 22]. Disponível em: https://www.ibge.gov.br/estatisticas/sociais/ saude/9160-pesquisa-nacional-de-saude.html?=\&t=downloads.

42. Programa das Nações Unidas para o Desenvolvimento. Ranking IDHM Municípios 2010; c2021 [acessado 2021 abr 22]. Disponível em: https://www. br.undp.org/content/brazil/pt/home/idh0/rankings/ idhm-municipios-2010.html.
Artigo apresentado em 12/02/2021

Aprovado em 09/06/2021

Versão final apresentada em 11/06/2021

Editores-chefes: Romeu Gomes, Antônio Augusto Moura da Silva 
